\title{
Peces arrecifales someros en el islote rocoso La Viuda, Golfo Dulce, Costa Rica
}

\author{
Gerardo Emigdio Palacios-Martínez
}

Fundación Proar, Liberia, Costa Rica; gerarp2003@hotmail.com, gerardopalacios2009@gmail.com

\author{
Recibido 11-VIII-2014. Corregido 20-XI-2014. Aceptado 22-XII-2014.
}

\begin{abstract}
Shallow reef fishes around "La Viuda" rocky islet, Golfo Dulce, Costa Rica. Rocky reefs maintain a high diversity of organisms. Differences in fish community could be expected by seasonal events and wave

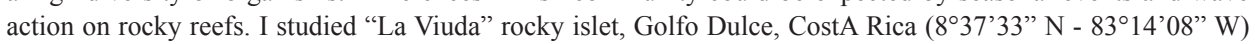
with Bohnsack and Bannerot visual census methods four times between February and December 2002. I identified 28 species and the most abundant families were Pomacentridae and Labridae. Shannon-Wiener diversity was between 1.1 and 1.73 nits/ind., and Pielou 0.43 . There is a higher diversity in the dry season and in areas protected from wave action. Rev. Biol. Trop. 63 (Suppl. 1): 329-338. Epub 2015 April 01.
\end{abstract}

Key words: fish community, rocky reefs, Golfo Dulce, visual census, $\mathrm{pH}$, temperature, Secchi disc, salinity.

Los arrecifes rocosos son ambientes que sustentan una alta diversidad de peces (Barjau, Rodríguez-Romero, Galván, Gutiérrez, \& López, 2012). Estas zonas se caracterizan por la alta energía liberada por oleaje y cambios de la marea que afectan la distribución de los organismos que habitan allí. La acción de las olas y los materiales acarreados por el agua, como arena, rocas y madera flotante, afectan la densidad de invertebrados y algas, dependiendo si ese lado de la roca está protegido o expuesto al oleaje (Levinton, 1995). Diferentes cambios estructurales en las comunidades ícticas dependen de factores físico químicos como la temperatura, la salinidad, el $\mathrm{pH}$ y la visibilidad (e.g. Tait, 1987; Arceo-Carranza \& Vega-Cendejas, 2009; Muñoz-Rojas, Ayala-Pérez, Sosa-López, \& Villalobos-Zapata, 2013). Allen y Robertson (1994) brindan información a nivel regional sobre la estructura de la comunidad íctica de los arrecifes rocosos e historia natural por especie de una gran variedad de peces a nivel del Pacífico Oriental Tropical. Por otro lado, en México, Pérez-España, Galván, y Abitia (1996) registran variaciones estructurales y temporales de la comunidad íctica, Jiménez-Gutiérrez (1999) estudió la abundancia y estructura comunitaria de peces en México y Galván-Villa, Arreola-Robles, Ríos-Jara, y Rodríguez-Zaragoza (2010) evaluaron los ensamblajes ícticos y su relación con el hábitat en México.

A nivel nacional, se tiene información sobre estructura íctica y ecología para el Pacífico Norte y Pacífico Sur costarricense (e.g. Espinoza \& Salas, 2005; Salas \& Alvarado, 2008).

Golfo Dulce es un pseudo fiordo cuyas características anóxicas le confieren condiciones atípicas para la región que junto al desarrollo agrícola, urbanístico, turístico y pesquero lo hacen muy vulnerable (e.g. Svendsen et al., 2006). Dentro del Golfo Dulce existen listados taxonómicos de peces, de las cuales 35 de un total de 71 especies registradas son no comerciales (e.g. Rojas-Figueroa, 2001; Salas \& Alvarado, 2008).

Los objetivos del presente estudio son: i) identificar las especies de peces arrecifales someras presentes en La Viuda y ii) determinar índices ecológicos relacionados con la 
abundancia y diversidad y iii) evaluar parámetros físico químicos del área de estudio.

\section{MATERIALES Y MÉTODOS}

Área de estudio: El Islote "Roca La Viuda" ( $\left.8^{\circ} 37^{\prime} 33^{\prime \prime} \mathrm{N}-83^{\circ} 14^{\prime} 08^{\prime \prime} \mathrm{W}\right)$ está ubicado en el Golfo Dulce, Costa Rica a 200m de la costa del océano Pacífico Oriental Tropical. Proviene de las estribaciones del Cerro de Nicuesa frente a punta Gallardo; en marea alta se cubre en su totalidad y en marea baja queda expuesto entre 1.5 y $2 \mathrm{~m}$, con una superficie expuesta aproximadamente de $6 \mathrm{~m}^{2}$, dependiendo de la marea. Algunos de los organismos que se encuentran en el arrecife son algas, plancton, cirripedios, anémonas, moluscos, octocorales, ofiuros y peces, esta diversidad se limita a los $10 \mathrm{~m}$ de profundidad en marea baja, por la interacción con los fondos suaves (obs. pers.).

Listado de especies, abundancia y diversidad: En febrero, abril, mayo y noviembre del 2002 se evaluaron los peces arrecifales someros de La Viuda en cuatro muestreos: Dos en época seca y dos en época lluviosa. Se aplicó el método de censos visuales de Bohnsack y Bannerot (1986) para determinar la diversidad y abundancia de peces. La Viuda se dividió en cuatro cuadrantes: el noreste, sureste, noroeste y suroeste, por medio de marcas geográficas en el campo, cada cuadrante se dividió en tres áreas iguales de $9 \mathrm{~m}^{2}$ para hacer cada censo por medio de una selección al azar. En cada censo, se identificó cada individuo hasta el taxón más bajo posible con la guía de peces de Allen y Robertson (1994). Se estimó la cantidad de individuos por especie que pasó en cada cuadrante de $9 \mathrm{~m}^{2}$ a profundidades de 0.5 y $10 \mathrm{~m}$ a lo largo de $10 \mathrm{~min}$ con tres réplicas al azar también delimitadas en campo con marcas geográficas, cuadrantes de aluminio, clavos y cuerdas. Posteriormente, se evaluaron los índices ecológicos de Sorensen $\left(\mathrm{I}_{\text {scual }}\right)$, índice de Shannon-Wiener $\left(\mathrm{H}^{\prime}\right)$, Índice de Pielou $\left(\mathrm{J}^{\prime}\right)$ y el índice de diversidad alfa de Margalef (IRE) (Villarreal et al., 2006).
Parámetros físico químicos: Desde junio del 2001 a noviembre del 2002 se midieron datos físico-químicos, en cada cuadrante de La Viuda a las profundidades de 0.5 y $10 \mathrm{~m}$. Se evaluó la temperatura del agua con un termómetro de alcohol $\left(-10\right.$ a $\left.100^{\circ} \mathrm{C}, 0.5\right)$. Se colectaron muestras de agua usando una Botella Niskin (1.5L) para medir la salinidad y $\mathrm{pH}$ en el laboratorio, por medio de un refractómetro (Nista ${ }^{\circledR}$ A 366 ATC, 0-100) y un pH-metro (Corning® $445,0.01$ ) y se determinó la visibilidad del agua con un Disco Secchi.

\section{RESULTADOS}

Listado de especies, abundancia y diversidad: A lo largo de todo el estudio se observaron 28 especies de peces someros de 18 familias en toda el área con un tiempo de estudio, total en época lluviosa de $30 \mathrm{~h}$ y en época seca fue de $30 \mathrm{~h}$. Con la intención de comparar la fauna íctica entre las dos épocas climáticas, se utilizó el índice de Shannon Weiner, que para la época seca fue de 1.73 nits/ind. y para la época lluviosa de 1.17 nits/ind (Cuadro 1). Este resultado es congruente con el IRE que en época seca fue de 3.52 y en la lluviosa de 2.85.

Ambas épocas climáticas presentaron a Thalassoma lucasanum (cabeciazul, Labridae) con la mayor abundancia relativa con $50 \%$ en la seca y $73 \%$ en la lluviosa, seguida de Chromis atrilobata (damisela, Pomacentridae) con $18 \%$ en la seca y $9 \%$ en la 1luviosa, Abudefduf trocschelii (damisela sargento, Pomacentridae) con 9\% y 5\% y Stegastes flavilatus (damisela, Pomacentridae) con $7 \%$ y $3 \%$ respectivamente, de las Familias Labridae y Pomacentridae (Fig. 1).

Nueve especies se encontraron en la época seca y no se presentaron en la lluviosa, mientras que seis especies de la lluviosa no se contabilizaron en la seca (Fig. 1).

Con respecto a la fauna íctica en los sectores expuestos y protegidos al oleaje en La Viuda, el índice de Shannon Weiner obtenido para la zona expuesta fue de 1.25 nits/ind. y en la zona protegida de 1.57 nits/ind (Cuadro 1). Este resultado es congruente con el IRE 
CUADRO 1

Índices ecológicos en La Viuda (LV) en Época seca (Es) y Época Lluviosa (EL1), entre Área expuesta (Ae) y Área protegida (Ap)

TABLE 1

Ecological index of compared aspects between La Viuda (LV) rocky isolet in Dry season (Es), and Wet season (ELl), and between Exposure area (Ae), and Protected area (Ap)

\begin{tabular}{lcccccc}
\multicolumn{1}{c}{ Aspecto } & $\mathrm{S}$ & $\mathrm{N}$ & $\mathrm{H}^{\prime}$ (nits/ind.) & $\mathrm{J}^{\prime}$ & IRE & Iscual \\
LV, Es $50 \mathrm{~m}^{2}$ & 18 & 125 & 1.73 & 0.6 & 3.52 & 0.38 \\
$\mathrm{LV}, \mathrm{ELl} 50 \mathrm{~m}^{2}$ & 15 & 136 & 1.17 & 0.43 & 2.85 & 0.38 \\
$\mathrm{LV}$, Ae $50 \mathrm{~m}^{2}$ & 11 & 64 & 1.25 & 0.52 & 2.4 & 0.52 \\
LV, Ap $50 \mathrm{~m}^{2}$ & 16 & 122 & 1.57 & 0.57 & 3.13 & 0.52 \\
$\mathrm{LV}, 50 \mathrm{~m}^{2}$ & 13 & 124 & 1.1 & 0.43 & 2.49 & 0.7
\end{tabular}

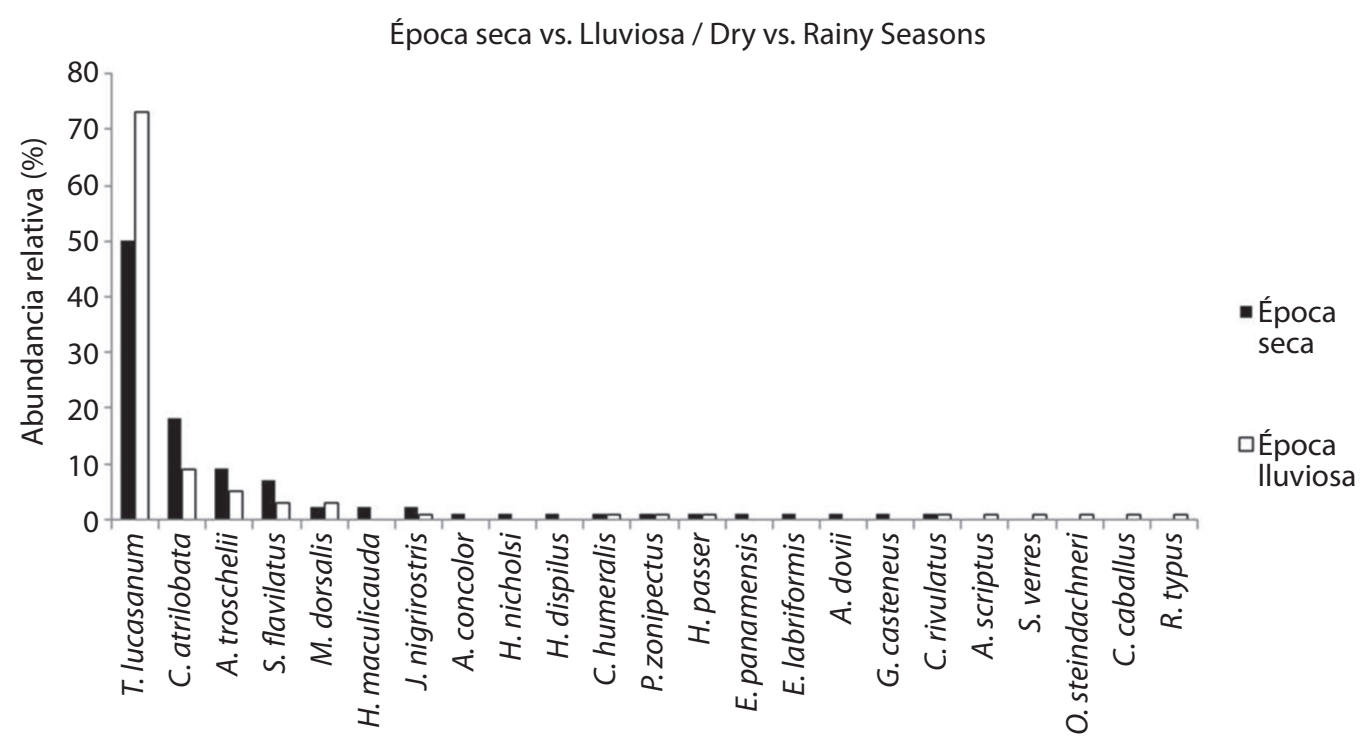

Fig. 1. Abundancias relativas de peces arrecifales en la época seca y lluviosa del 2002 en islote La Viuda, Golfo Dulce.

Fig. 1. Relative abundances of rocky reef fishes during dry 2002 and wet season around "La Viuda" rocky islet, Golfo Dulce.

que en la zona expuesta fue de 2.4 y en la protegida de 3.13 .

Ambas zonas presentaron a T. lucasanum con la abundancia relativa más alta con $69 \%$ en la expuesta y $55 \%$ en la protegida, seguida de C. atrilobata con $2 \%$ y $19 \%$, A.trocschelii con $9 \%$ y $6 \%$ y $S$. flavilatus con $5 \%$ y $8 \%$ respectivamente. Estas especies pertenecen a las familias Labridae y Pomacentridae (Fig. 2).

Dos especies se registraron en la zona expuesta y no se contabilizaron en la protegida y siete especies de la protegida no se presentaron en la expuesta (Cuadro 2).

Parámetros físico químicos: Los datos físicos-químicos evaluados en La Viuda, como el $\mathrm{pH}$ y la temperatura presentaron valores inversamente proporcionales a la profundidad, mientras que la salinidad era directamente proporcional. Las medidas de $\mathrm{pH}$ están en un rango entre 8.03-8.38, los de temperatura entre $29^{\circ} \mathrm{C}$ y $32.4^{\circ} \mathrm{C}$, la visibilidad del disco Secchi 


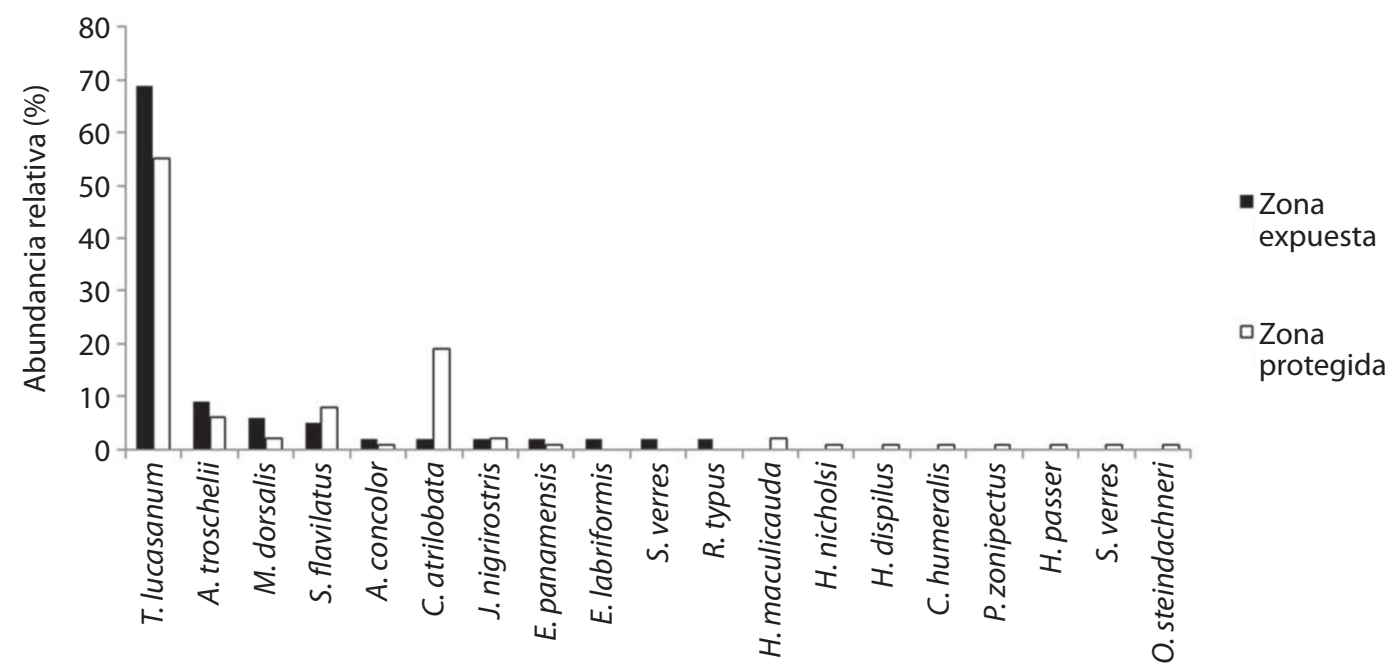

Fig. 2. Abundancias relativas de peces arrecifales en la zona expuesta y protegida del 2002 en islote La Viuda, Golfo Dulce. Fig. 2. Relative abundances of rocky reef fishes in exposure and protected areas from wave action around "La Viuda" rocky islet, Golfo Dulce.

\section{CUADRO 2}

Especies y familias de peces arrecifales registrados en La Viuda ${ }^{1}\left({ }^{*}\right.$ especies cuantificadas fuera de los censos visuales, seca $^{2}, \operatorname{lluviosa}^{3}$, expuesta ${ }^{4}$, protegida $^{5}$ )

TABLE 2

Shallow rocky fish species and families recorded in La Viuda ${ }^{1}$ ( ${ }^{*}$ Species quantified out visual census, dry season ${ }^{2}$, wet season ${ }^{3}$, exposure from wave action ${ }^{4}$, protected from wave action ${ }^{5}$ )

\begin{tabular}{|c|c|c|c|}
\hline Familia & Especie & Familia & Especie \\
\hline \multirow[t]{5}{*}{ Pomacentridae } & Abudefduf concolor ${ }^{1,2,4,5}$ & Muraenidae & Gymmothorax casteneus ${ }^{1,2}$ \\
\hline & Abudefduf troschelii ${ }^{1,2,3,4,5}$ & Haemulidae & Haemulon maculicauda ${ }^{2}$ \\
\hline & Chromis atrilobata $1,2,3,4,5$ & & Haemulon sexfasciatum* \\
\hline & Microspathodon dorsalis ${ }^{1,2,3,4,5}$ & Labridae & Halichoeres dispilus $^{2}$ \\
\hline & Stegastes $\mathrm{spp}^{1,2,3,4,5}$ & & Halichoeres nicholsi ${ }^{1,2}$ \\
\hline Chaenopsidae & Acanthemblemaria hancocki* & & Thalassoma lucasanum $^{1,2,3,4,5}$ \\
\hline Monacanthidae & Aluterus scriptus $^{3}$ & Pomacanthidae & Holacanthus passer ${ }^{1,2,3}$ \\
\hline Apogonidae & Apogon dovii ${ }^{1,2}$ & & Pomacanthus zonipectus $^{2,3}$ \\
\hline Gobiidae & Bathygobius sp* & Cirrhitidae & Cirrhitus rivulatus $^{1,2,3,4}$ \\
\hline Carangidae & Caranx caballus ${ }^{3}$ & Blenniidae & Ophioblennius steindachneri ${ }^{3}$ \\
\hline \multirow[t]{2}{*}{ Chaetodontidae } & Chaetodon humeralis ${ }^{2,3}$ & Rhincodontidae & Rhincodon typus ${ }^{3}$ \\
\hline & Johnrandallia nigrirostris ${ }^{1,2,3,4,5}$ & Scaridae & Scarus rubroviolaceus $*$ \\
\hline \multirow[t]{2}{*}{ Serranidae } & Epinephelus labriformis ${ }^{2}$ & Balistidae & Sufflamen verres ${ }^{3}$ \\
\hline & Cephalopholis panamensis ${ }^{1,2,4,5}$ & Zanclidae & Zanclus cornutus* \\
\hline Familias totales 18 & & Especies totales 28 & \\
\hline
\end{tabular}


entre 2.5 y $14 \mathrm{~m}$ y los rangos de salinidad se encuentran entre 27.8-33.0 (Fig. 3).

\section{DISCUSIÓN}

Listado de especies, abundancia y diversidad: La riqueza total de este estudió llegó a 28 especies lo cual es el $10 \%$ de la riqueza publicada para los arrecifes rocosos del Golfo de California (Barjau et al., 2012), riqueza que se asume como representativa, ya que una comunidad de peces arrecifales rocosos se estabiliza en 23 especies hasta profundidades entre 50 y 60m (Jiménez-Gutiérrez, 1999). Esto no sucede en el promedio de las riquezas de cada tratamiento, el cual se ubicó entre 11 y 18 especies, lo cual podría explicarse a que este estudio sólo incluyó un área geográfica de alrededor de $50 \mathrm{~m}^{2}$ en total, con una profundidad máxima de $10 \mathrm{~m}$.

Dentro de los estudios realizados en el país y que llevó a cabo la misma metodología, está el de Schaper (1996) quien realizó el muestreo en el Caribe entre enero y marzo de 1994 sin detallar el esfuerzo de muestreo y este estudio se realizó en el Pacífico en los meses de Febrero, Abril, Mayo y Noviembre del 2002 para un total de 60 horas de muestreo, Schaper (1996) encontró una diversidad de 54 especies de peces en el arrecife coralino de Puerto Viejo; comparado con las 28 especies resultantes de este trabajo. Este resultado podría explicarse por las diferencias en el área de muestreo, rugosidad y profundidad. Además, el estudio de Schaper (1996) incluyó mayoritariamente a especies permanentes, es decir especies que se encontraron en todos los muestreos, con bajas o mínimas observaciones de las especies estacionales, es decir aquellas que usan el hábitat temporalmente o las crípticas, aquellas especies que viven en grietas, lo que acentúa la diferencia de la riqueza total.

Las diversidades $\left(\mathrm{H}^{\prime}\right)$ de este estudio se encuentran entre 1.1 hasta 1.73 , valores por debajo a las diversidades encontradas por Schaper (1996) en Puerto Viejo, las cuales están entre 1.94 y 2.28. Las diversidades encontradas en Chávez (2009) son más altas que las encontradas en este estudio, pero las diversidades de La Viuda se encuentran dentro del
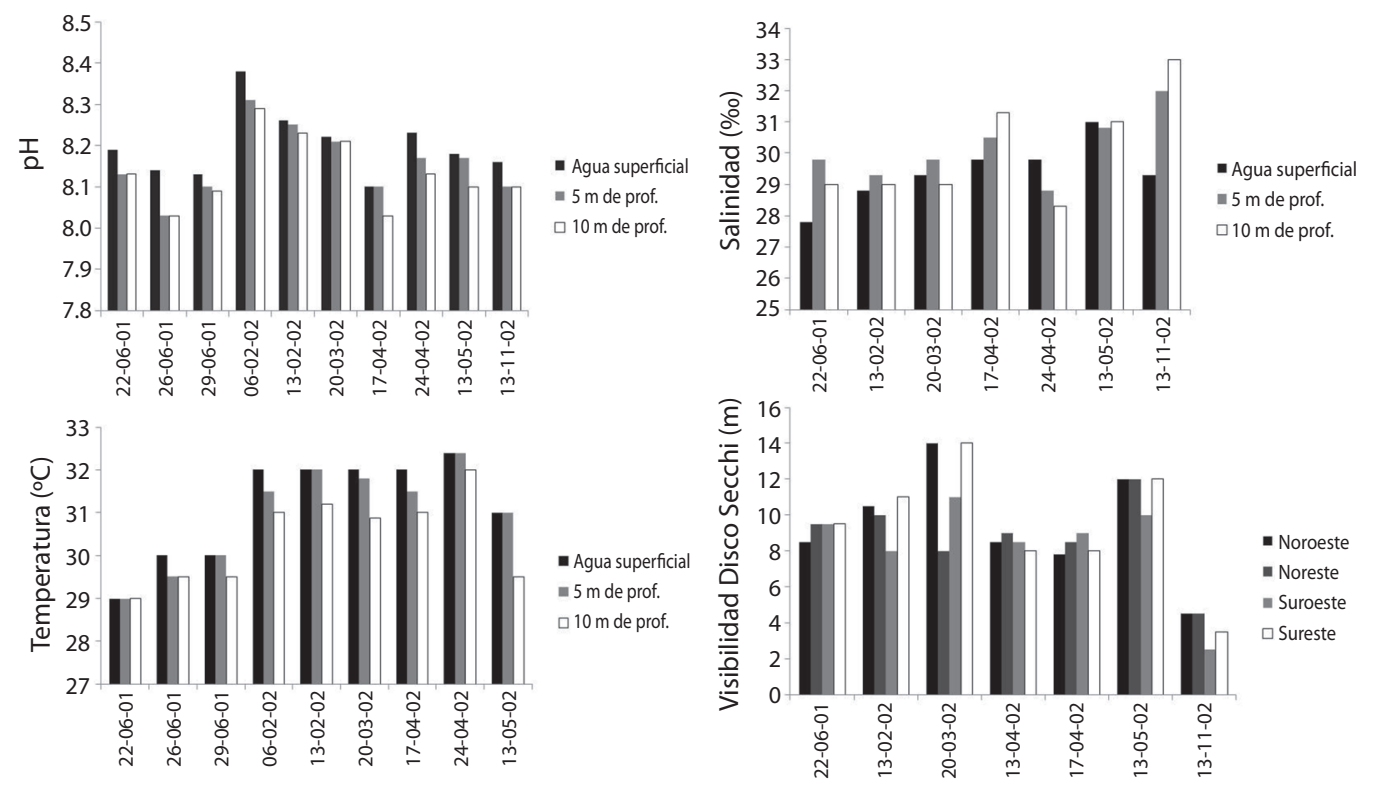

Fig. 3. Datos físicos-químicos medidos desde el 2001 al 2002 en La Viuda, Golfo Dulce.

Fig. 3. Physico-chemical data measured from 2001 to 2002 around La Viuda" rocky islet, Golfo Dulce. 
rango registrado en Islas Galápagos entre 1.1 y 3.1bits/ind. (Margalef, 1981); haciendo la salvedad que en Ecuador, los valores de diversidad se calcularon con "base n" pero se indica en bits/ind., en este estudio se calculó con base $\mathrm{n}$ pero se anota como nits/ind. para diferenciarla con las de base dos y diez. Por otro lado, los valores más altos de este estudio coinciden con los valores más bajos de diversidad de PérezEspaña et al. (1996) (Cuadro 3).

Las diversidades de La Viuda son inferiores comparadas con Barjau et al. (2012) y Palacios-Salgado (2005), lo cual se explica por las diferencias geográficas, la exclusión de especies crípticas y temporales, la metodología usada y diferencias en esfuerzo y métodos de muestreo (Cuadro 3).

En todos los tratamientos las especies más abundantes fueron T. lucasanum, A. trocschelii, C. atrilobata, y $S$. spp, lo que coincide con el estudio de Pérez-España et al. (1996). Las tres primeras especies coincide con Barjau et al. (2012), mientras que $S$. spp no se encuentra tan bien representada como si sucede en este estudio. La coincidencia de especies en general es común, ya que todas tienen una distribución desde el Golfo de California hasta al menos Panamá o Perú (Allen \& Robertson, 1994). Por otro lado, Salas y Alvarado (2008) registran a T. lucasanum, S. acapulcoensis (Damisela, Pomacentridae) y S. flavilatus como las más abundantes en el Pacífico central costarricense, al igual que este estudio, sin incluir a C. atrilobata, únicamente lo hacen en un caso local, mientras que en esta investigación se presentó en todos los tratamientos. Estos autores discuten la presencia abundante de T. lucasanum por la cobertura de corales duros, lo que difiere de esta investigación, ya que estos ecosistemas rocosos presentaron una presencia baja de corales duros comparada con la abundancia de octocorales como Carijoa sp y Leptogorgia alba. La alta abundancia de T. lucasanum en el presente estudio podría explicarse a que los meses de muestreo coincidieron con hembras activamente reproductivas que se ven influenciadas a lo largo del año por el ciclo de mareas (Warner, 1982); sin embargo, se necesitan estudios de mayor esfuerzo para explicar la abundancia de T. lucasanum, S. acapulcoensis y S. flavilatus en La Viuda.

En todos los tratamientos se vieron cambios en la diversidad, lo que coincide con las observaciones realizadas por Barjau et al. (2012). Estas especies pueden vivir en diferentes hábitats por que se alimentan de una amplia variedad de flora y fauna, además son frecuentes a bajas profundidades (Allen \& Robertson, 1994). Las familias Labridae y Pomacentridae presentan las especies con mayor abundancia relativa en este estudio, lo que es común en arrecifes rocosos (Perez-Cruet \& Phillips, 1983; Espinoza \& Salas, 2005; Chávez, 2009; Galván-Villa et al., 2010). Este estudio coincide con las observaciones de Chávez (2009) y Jiménez-Gutiérrez (1999), los cuales identifican a $C$. atrilobata y a $T$. lucasanum como entre las más abundantes, pero difiere en que este estudio encuentra más abundante a T. lucasanum que a $C$. atrilobata.

La presencia de especies como Chaetodon humeralis (Mariposa, Chaetodontidae), Johnrandallia nigrirostris (Mariposa dorada, Chaetodontidae), H. maculicauda (Roncador, Haemulidae), E. labriformis (Mero, Serranidae) y C. panamensis (Mero, Serranidae) en La Viuda podría indicar que se encuentre en buenas condiciones de salud, ya que presentan hábitos coralívoros y si se presenta algún estrés ambiental que reduzca estas poblaciones de celenterados, esta comunidad íctica tiende a desplazarse para encontrar arrecifes en buen estado (Chávez, 2009).

Al comparar la fauna íctica somera en dos épocas climáticas en La Viuda, al igual que lo discute Galván-Villa et al. (2010), se observa como el índice de diversidad $\left(\mathrm{H}^{\prime}\right)$ de ambas épocas presenta diferencias. Este estudio encontró 0.56 nits/ind. de diferencia entre ambas épocas climáticas, donde 16 especies diferentes se presentaron en una época y no en la otra, lo que indica que posiblemente la época climática tiene un efecto entre las comunidades ícticas someras de arrecifes rocosos.

La época seca presentó mayor diversidad, mayor equidad y mayor riqueza que la época 


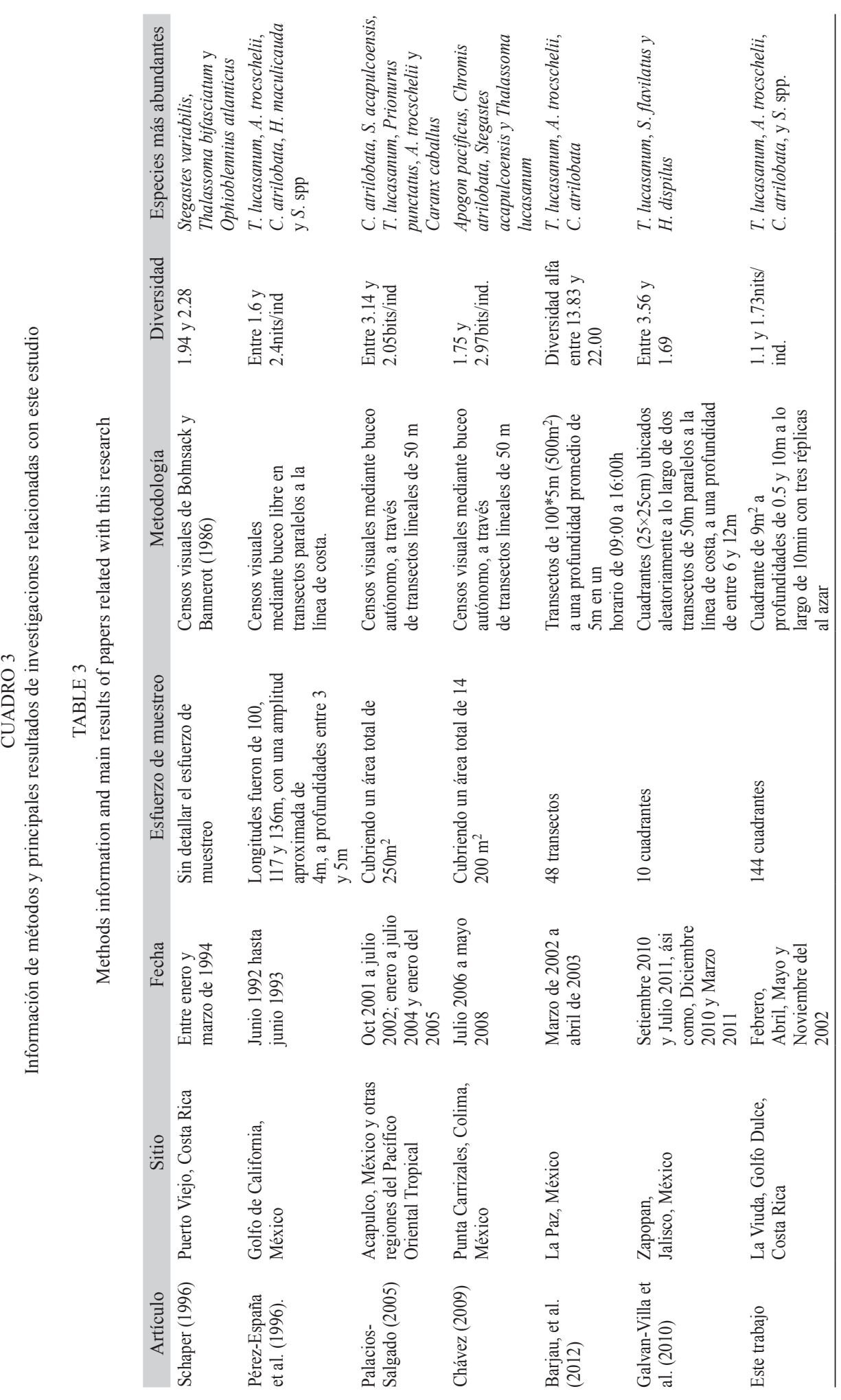

(c) $\fallingdotseq$ Rev. Biol. Trop. (Int. J. Trop. Biol. ISSN-0034-7744) Vol. 63 (Suppl. 1): 329-338, April 2015 
lluviosa, coincidiendo con los estudios de Pérez-España et al. (1996), Krebs (1998), Jiménez-Gutiérrez (1999) y Barjau et al. (2012), los cuales encontraron cambios en las diversidades, riquezas y abundancias ícticas con las época climáticas y lo explican por las diferencias de la temperatura del agua en latitudes tropicales y subtropicales, régimen de lluvias, tipo de hábitat, profundidad, corrientes, surgencias, giros ciclónicos y fauna y flora circundantes.

Este estudio presentó mayor número de individuos en la época lluviosa que en la época seca. Sin embargo, no siempre el régimen de lluvias causa los mismos efectos en la estructura íctica de los diferentes arrecifes, aspecto que debe relacionarse con la intensidad y temporalidad de las mismas (Palacios-Salgado, 2005), además del limitado esfuerzo de muestreo para el presente caso.

Para la fauna íctica en los sectores expuestos y protegidos al oleaje en La Viuda, el área expuesta presentó menor diversidad, equidad y riqueza de especies ícticas que el área protegida, de acuerdo a los índices ecológicos analizados, esta misma condición la encontró Galván-Villa et al. (2010). Estas diferencias entre riquezas y abundancias se explican por la disponibilidad de alimento y por el reclutamiento de especies en cada sitio (Barjau et al., 2012). En La Viuda, los peces permanentes dependen de la densidad de su base alimenticia, principalmente invertebrados y algas (Allen \& Robertson, 1994; Pérez-España et al., 1996; Schaper, 1996; Arreola-Robles, 1998; Jiménez-Gutiérrez, 1999; Galván-Villa et al., 2010), los cuales presentan mayores abundancias en la zona protegida al oleaje (Allen \& Robertson, 1994; Pérez-España et al., 1996; Schaper, 1996; Arreola-Robles, 1998; JiménezGutiérrez, 1999; Galván-Villa et al., 2010), por ende, los peces se concentran más allí. Estas variaciones también se explican por las características del sustrato, profundidad, visibilidad, corrientes, acción del oleaje y el viento (Allen \& Robertson, 1994; Pérez-España et al., 1996; Schaper, 1996; Arreola-Robles, 1998; JiménezGutiérrez, 1999; Galván-Villa et al., 2010).
Parámetros físico químicos: Los datos físicos-químicos evaluados se encuentran entre los rangos normales para el área (Richards, Anderson, \& Cline, 1971; Córdoba \& Vargas, 1996; Hossfeld, 1996); Entre las diferencias encontradas en los índices ecológicos calculados en esta investigación, están los cambios entre la época seca y la lluviosa, principalmente de la temperatura, la salinidad, el $\mathrm{pH}$ y la visibilidad. Se recomienda hacer un seguimiento continuo de estos aspectos para poder establecer relaciones entre la estructura de la comunidad íctica y el cambio climático global.

Con respecto al $\mathrm{pH}$ este estudio midió valores entre 8.38 y 8.03 , lo que coincide con Richards et al. (1971) con un pH entre 8.4 y 7.9 decreciendo con la profundidad, como también se registró en este trabajo.

Esta investigación determinó temperaturas entre $29^{\circ} \mathrm{C}$ y $32.4^{\circ} \mathrm{C}$ a los 0,5 y $10 \mathrm{~m}$ temperaturas normales para la zona, coincidiendo con Córdoba y Vargas (1996) con $31.5^{\circ} \mathrm{C}$ a $10 \mathrm{~m}$ de profundidad, el 12 de enero de 1994; Hossfeld (1996) documentó el 8 de febrero de 1994, $29.7^{\circ} \mathrm{C}$ en agua superficial en la estación 02 , la más costera. Richards et al. (1971) registró $27.54^{\circ} \mathrm{C}$ en la estación cinco, la más costera, a $10 \mathrm{~m}$ de profundidad, a $5 \mathrm{~m}$ de profundidad con $28.77^{\circ} \mathrm{C}$ y a $0 \mathrm{~m}$ con $32.26^{\circ} \mathrm{C}$.

La salinidad se encontró entre 27.8-33.0 entre 0 y $10 \mathrm{~m}$ de profundidad, rango que incluye los datos registrados por Córdoba y Vargas (1996) con una salinidad de 29.5 a $0 \mathrm{~m}$, los de Hossfeld (1996) con 28.5-29.7 en la superficie y el de Richards et al. (1971) con un rango entre $31.69-32.51$ a 0 y $10 \mathrm{~m}$ de profundidad.

Como conclusiones, este estudió determinó que se pueden hacer estimaciones de diversidad y abundancia de peces arrecifales someros a lo largo del año con el método de censos visuales. Las diversidades encontradas en La Viuda se encuentran dentro del rango registrado en Islas Galápagos entre 1.1 y 3.1bits/ind. Las especies más abundantes fueron T. lucasanum, A. trocschelii, C. atrilobata, y $S$. spp, de las familias Labridae y Pomacentridae. De acuerdo a los índices ecológicos se encontraron diferencias entre las comunidades 
ícticas someras de arrecifes rocosos, debido a las épocas climáticas y a la acción del oleaje.

\section{AGRADECIMIENTOS}

Agradezco al Instituto de Estudios Tropicales y a la Universidad de Golfito por proporcionarme el apoyo financiero para la fase de campo de este estudio, así como al

PROMACOSA-SINAC, por darme las facilidades para completar este esfuerzo.

\section{RESUMEN}

Los arrecifes rocosos proveen una alta diversidad de organismos. Se presentaron cambios en la estructura de la comunidad íctica a lo largo de la estación climática y la acción del oleaje. Este estudio evaluó la diversidad y la abundancia de los peces del arrecife rocoso La Viuda, Golfo Dulce, Costa Rica ( $8^{\circ} 37^{\prime} 33^{\prime \prime} \mathrm{N}-8^{\circ} 14^{\prime} 08^{\prime}$ 'W). Los peces fueron estudiados con el método de Bohnsack y Bannerot comparando las estaciones seca y lluviosa, las zonas protegidas y expuestas al oleaje usando índices ecológicos. El total de especies fue 28, las familias más abundantes fueron Pomacentridae y Labridae. La diversidad de Shannon-Wiener resultó entre 1,1 y 1,73 nits/ ind. El índice de Pielou en La Viuda fue 0.43. Se encontró mayor diversidad de especies en la época seca y en la zona protegida al oleaje.

Palabras clave: comunidad íctica, arrecife rocoso, Golfo Dulce, censo visual, $\mathrm{pH}$, temperatura, disco Secchi, salinidad.

\section{REFERENCIAS}

Allen, G. R. \& Robertson, D. R. (1994). Fishes of the Tropical Eastern Pacific. USA: University of Hawii Press.

Arceo-Carranza, D. \& Vega-Cendejas, M. E. (2009). Spatial and temporal characterization of fish assemblages in a tropical coastal system influenced by freshwater inputs: northwestern Yucatan peninsula. Revista de Biología Tropical, 57(1-2), 89-103.

Arreola-Robles, J. L. (1998). Diversidad de peces de arrecife en la región de La Paz, B.C.S., México (Tesis de Maestría). CICIMAR-IPN, La Paz, B.C.S., México.

Barjau, E., Rodríguez-Romero, J., Galván, F., Gutiérrez, F., \& López, J. (2012). Estructura temporal y espacial de la comunidad de peces arrecifales de la Isla San José, Golfo de California, México. Revista de Biología Tropical, 60(2), 649-667.
Bohnsack, J. A. \& Bannerot, S. P. (1986). A stationary visual census technique for quatitatively assessing community structure of coral reef fishes (NOAA Technical Report NMFS 41). USA: Department of Comerce.

Chávez Comparán, J. C. (2009). Caracterización y estructura de la ictiofauna de arrecifes rocosos y coralinos en Punta Carrizal, Colima, México para fines de inventario y conservación de la diversidad animal (Proyecto No. DJ020, Informe final SNIBCONABIO). D. F., México: Universidad de Colima.

Córdoba, R. \& Vargas, J. (1996). Temperature, salinity, oxygen, and nutrient profiles at a $200 \mathrm{~m}$ deep station in Golfo Dulce, Pacific coast of Costa Rica. Revista de Biología Tropical, 44(3), 233-236.

Espinoza, M. \& Salas, E. (2005). Estructura de las comunidades de peces de arrecife en las Islas Catalinas y Playa Ocotal, Pacífico Norte de Costa Rica. Revista de Biología Tropical, 53, 523-536.

Galván-Villa, C. M., Arreola-Robles, J. L., Ríos-Jara, E., \& Rodríguez-Zaragoza, F. A. (2010). Ensamblajes de peces arrecifales y su relacion con el habitat bentónico de la Isla Isabel, Nayarit, México. Revista de Biología Marina y Oceanografia, 45, 311-324.

Hossfeld, B. (1996). Distribution and biomass of arrow worms (Chaetognatha) in Golfo de Nicoya and Golfo Dulce, Costa Rica. Revista de Biología Tropical, 44(Suppl. 3), 157-172.

Jiménez-Gutiérrez, S. (1999). Abundancia y estructura comunitaria de peces de arrecife rocoso en la zona de isla cerralvo, B.C.S., México (Tesis de maestría). CICIMAR, México.

Krebs, C. (1998). Ecological Methodology. USA: Benhamin-Cummings/Wesley Longman, Inc.

Levinton, J. S. (1995). Marine Biology: Function, Biodiversity, Ecology. New York, USA: Oxford University Press.

Margalef, D. R. (1981). Ecología. España: Omega.

Muñoz-Rojas, S., Ayala-Pérez, L. A., Sosa-López, A., \& Villalobos-Zapata, G. J. (2013). Distribución y abundancia de la comunidad de peces en la porción litoral de la Reserva de la Biósfera Los Petenes, Campeche, México. Revista de Biología Tropical, 61(1), 213-227.

Palacios-Salgado, D. S. (2005). Asociaciones de peces en los arrecifes rocosos de la Bahía de Acapulco, Guerrero, México (Tesis de Maestría). CICIMAR-IPN, La Paz B.C.S., México.

Perez-Cruet, M. J. \& Phillips, P. C. (1983). A comparative survey of reef fishes in Caribbean and Pacific Costa Rica. Revista de Biología Tropical, 32(1), 95-102.

Pérez-España, E. H., Galván, M. F., \& Abitia, C. A. (1996). Variaciones temporales y espaciales en la estructura de la comunidad de peces de arrecifes rocosos del 
suroeste del Golfo de California, México. Ciencias Marinas, 22(3), 273-294.

Richards, F. A., Anderson, J. J., \& Cline, J. D. (1971). Chemical and physical observations in Golfo Dulce, an anoxic basin on the pacific coast of Costa Rica. Limnology and Oceanography, 16(1), 43-50.

Rojas-Figueroa, R. (2001). Caracterización de la ictiofauna de los sustratos duros de la parte interna del Golfo Dulce, Costa Rica. (Tesis de licenciatura). Escuela de Biología, Universidad de Costa Rica, Costa Rica.

Salas, E. \& Alvarado, J. (2008). Lista de peces costeros del Parque Nacional Marino Ballena, Costa Rica, con anotaciones sobre su ecología. Brenesia, 69, 43-58.

Schaper, S. (1996). La Comunidad de Peces en el Arrecife de Puerto Viejo (Limón, Costa Rica). Revista de Biología Tropical, 44(2), 923-925.
Svendsen, H., Rosland, R., Myking, S., Vargas, J. A. Lizano, O., \& Alfaro, E. (2006). A physical-oceanographic study of Golfo Dulce, Costa Rica. Revista de Biología Tropical, 54(Suppl. 1), 147-170.

Tait, R. (1987). Elementos de ecología marina. España: Edit. Acribia.

Villarreal H., Álvarez, M., Córdoba, S., Escobar, F., Fagua, G., Gast, F., ... Umaña, A. M. (2006). Manual de métodos para el desarrollo de inventarios de biodiversidad, Programa de Inventarios de Biodiversidad. Bogotá, Colombia: Instituto de Investigación de Recursos Biológicos Alexander von Humboldt.

Warner, R. R. (1982). Mating systems, sex change and sexual demography in the rainbow wrasse, Thalassoma lucasanum. Copeia, 3, 653-661. 